IPM/P-2012/050

\title{
On Holography with Hyperscaling Violation
}

\author{
Mohsen Alishahiha $^{a}$, Hossein Yavartanoo ${ }^{b}$ \\ a School of physics, Institute for Research in Fundamental Sciences (IPM) \\ P.O. Box 19395-5531, Tehran, Iran \\ ${ }^{b}$ Department of Physics, Kyung-Hee University, Seoul 130-701, Korea \\ E-mails: alishah@ipm.ir, yavar@khu.ac.kr
}

\begin{abstract}
We study certain features of strongly coupled theories with hyperscaling violation by making use of their gravitational duals. We will consider models with an anisotropic scaling in time or in one of spatial directions. In particular for the case where the anisotropic scaling is along a spatial direction we will compute the holographic entanglement entropy and show that for specific values of the parameters it exhibits a logarithmic violation of the area law. We will also probe the backgrounds by different closed and open strings which in turn can be used to read, for example, effective potential of an external object, drag force and etc.
\end{abstract}




\section{Introduction}

AdS/CFT correspondence [1] has thought us to think of a gravitational theory on an asymptotically locally AdS geometry as a dual description of a strongly coupled field theory with a UV fixed point. It is dual in the sense that there is a one to one correspondence between objects in the gravitational theory and those in the dual field theory [2,3]. Having an AdS geometry guarantees that the dual field theory has conformal symmetry.

It is always challenging to see in what extend the gauge/gravity correspondence can be generalized to geometries which are not asymptotically AdS. In particular one may consider the case where the theory is not conformally invariant, though it may still be invariant under a certain scaling. Indeed such a generalization, has been made in literature in the context of the application of the AdS/CFT correspondence in the condensed matter physics (for a review see for example [4]). A prototype example of such a model is a theory with the Lifshitz fixed point where the theory has a spatial isotropic scale invariance which is characterized by a dynamical exponent, $z$, as follows

$$
t \rightarrow \lambda^{z} t, \quad x_{i} \rightarrow \lambda x_{i},
$$

where $t$ is time and $x_{i}$ s are spatial directions of the space-time.

The holographic description of a $(d+1)$-dimensional theory with the Lifshitz fixed point has been considered in $[5]$ where it was proposed that the corresponding gravitational dual can be defined by a gravity on the following metric (see also [7]) $)^{2}$

$$
d s_{d+2}^{2}=R^{2}\left(-\frac{d t^{2}}{r^{2 z}}+\frac{d r^{2}}{r^{2}}+\frac{\sum_{i=1}^{d} d x_{i}^{2}}{r^{2}}\right),
$$

where $R$ is the radius of curvature ${ }^{3}$

It is worth to mention that a metric with Lifshitz isometry is not a solution of a pure cosmological Einstein gravity. This is simply because in the pure Einstein gravity there is nothing to produce an anisotropic in the space-time. In fact to obtain such a solution one needs to couple the Einstein gravity to other fields. In the minimal case the extra field could be a massive gauge field [7] which can, indeed, produce an anisotropic in the space-time leading to Lifshitz geometry.

More naturally the Lifshitz metric may be found in an Einstein-Maxwell-Dilaton theory $[11-16]$. Actually by including both dilaton (in general a scalar with nontrivial potential) and an abelian gauge field, one can find even more interesting

\footnotetext{
${ }^{1}$ See also $[6]$ for an earlier work on a geometry with the Lifshitz scaling.

${ }^{2}$ Geometries with non-relativistic conformal symmetry have also been considered in [8,9]. For earlier work on geometry with Schrödinger symmetry see 10 .

${ }^{3}$ Through out this paper we set $R=1$.
} 
metrics. In particular one may obtain a metric with the following form [17]

$$
d s_{d+2}^{2}=r^{\frac{2 \theta}{d}}\left(-\frac{d t^{2}}{r^{2 z}}+\frac{d r^{2}}{r^{2}}+\frac{\sum_{i=1}^{d} d x_{i}^{2}}{r^{2}}\right),
$$

where the constants $z$ and $\theta$ are dynamical and hyperscaling violation exponents, respectively. This is the most general geometry which is spatially homogeneous and covariant under the following scale transformations

$$
t \rightarrow \lambda^{z} t, \quad r \rightarrow \lambda r, \quad x_{i} \rightarrow \lambda x_{i}, \quad d s_{d+2} \rightarrow \lambda^{\frac{\theta}{d}} d s_{d+2}
$$

Note that the metric (1.3) is conformally a Lifshitz geometry which is the scale invariant limit $\theta=0$ of $(1.3)$. Indeed with a non-zero $\theta$, the distance is not invariant under the scaling which in the context of AdS/CFT indicates violations of hyperscaling in the dual field theory. More precisely, while in $(d+1)$-dimensional theories without hyperscaling (dual to background $(1.2)$ ) the entropy scales as $T^{d / z}$ with temperature, in the present case (dual to background (1.3)) it scales as $T^{(d-\theta) / z}$ [18, 19.

Holographic aspects of theories with hyperscaling violation have been studied in [20] where the authors have shown that in order to have a physically sensible dual field theory one should assume

$$
(d-\theta)(d z-\theta-d) \geq 0, \quad(z-1)(d+z-\theta) \geq 0
$$

which is a necessary condition for the null energy condition to be satisfied, i.e. $T_{\mu \nu} N^{\mu} N^{\nu} \geq 0$. In particular it was shown that for $\theta=d-1$ the holographic entanglement entropy shows a logarithmic violation of the area law indicating that the dual theory exhibits an $\mathcal{O}\left(N^{2}\right)$ Fermi surface 19,21]. Therefore the geometry (1.3) with specific values of its parameters could provide a gravitational dual for a system with Fermi surface in any dimensions.

However, we note that due to hyperscaling violation as well as the behavior of dilaton field at large distances, the background (1.3) cannot provide a dual description of a theory in all range of energies from UV to IR [22, 23] (see also [20]) . In fact gravity on the background (1.3) may be considered as an effective theory valid over an intermediate energy scale.

One of the aim of the present paper is to further explore certain features of hyperscaling violation theories by making use of the gravity dual given by (1.3). More precisely we will probe the background by an open string which in turns can be used to study the response of the theory to an external object. In particular we shall study potential between external objects, drag force as well as a rotating object. We will also make a comment on a closed string probing the background.

Following [26] we will also consider a geometry with an anisotropic scaling in a spatial direction. The corresponding geometry can be obtained by a double Wick rotation from the metric (1.3). To be more concrete, consider a double Wick rotation 
as follows

$$
t \rightarrow i y, \quad x_{d} \rightarrow i t
$$

then the metric 1.3 becomes

$$
d s_{d+2}^{2}=r^{\frac{2 \theta}{d}}\left(\frac{d y^{2}}{r^{2 z}}+\frac{d r^{2}}{r^{2}}+\frac{\sum_{i=1}^{d-1} d x_{i}^{2}}{r^{2}}-\frac{d t^{2}}{r^{2}}\right) .
$$

The above metric is covariant under the following scale transformations

$$
y \rightarrow \lambda^{z} y, \quad r \rightarrow \lambda r, \quad d s_{d+2} \rightarrow \lambda^{\frac{\theta}{d}} d s_{d+2}, \quad\left(t, x_{i}\right) \rightarrow \lambda\left(t, x_{i}\right),
$$

for $i=1, \cdots, d-1$.

In the present paper we will also intend to study holographic aspects a theory whose dual gravitational theory is given by a gravity on the background (1.7). In particular, we study holographic entanglement entropy in this background. We show that for $\theta=d-1$ for any $z$, the entanglement entropy exhibits a logarithmic violation of the area law showing that the geometry may also provide a suitable gravitational description for theories with $\mathcal{O}\left(N^{2}\right)$ Fermi surface. We will also probe this geometry by an open string.

The paper is organized as follows. In the next section we study holographic entanglement entropy using the metric (1.7). In section three we will consider an open string probing the metrics (1.3) and (1.7). For completeness of our study we will consider a closed string probe in section four. The last section is devoted to discussions. In what follows we only consider the case with $\theta \leq d$.

\section{Holographic entanglement entropy}

In this section we will study the entanglement entropy by making use of the AdS/CFT correspondence. To compute the entanglement entropy via AdS/CFT correspondence one needs to minimize a surface in the bulk gravity. More precisely, given a gravitational theory with the bulk Newton's constant $G_{N}$, the holographic entanglement entropy is given by 24,25

$$
S_{A}=\frac{\operatorname{Area}\left(\gamma_{A}\right)}{4 G_{N}},
$$

where $\gamma_{A}$ is the minimal surface in the bulk whose boundary coincides with the boundary of the entangling region.

It is important to note that in order to compute the holographic entropy, since we are dealing with a spatial surface, one needs to work at constant time. In other words, the $g_{t t}$ component of the metric does not, directly, contribute to the holographic entanglement entropy. On the other hand, for the hyperscaling violating geometry (1.3), there are non-trivial effects due to non-relativistic nature of the 
metric which in turn comes from the $g_{t t}$ component.

Holographic entanglement entropy of the background $(1.3)$ has been studied in [20] where it was shown that the entanglement entropy exhibits interesting behaviors for $d-1 \leq \theta \leq d$. Indeed while the theory has extension entropy for $\theta=d$ it shows logarithmic violation of area law for $\theta=d-1$.

In this section following [26] we would like to study holographic entanglement entropy for the background (1.7). For this metric even at constant time slices the metric has an anisotropic scaling.

To proceed, we will calculate entanglement entropy for a strip subsystem in the dual theory. From gravity point of view one needs to minimize a surface in the geometry (1.7) whose intersection with the boundary coincides to the strip. In course to do so, we recognize two possible ways to embed the strip in the theory depending on the directions we choose for the strip. In the first case the width of the strip is along $y$ direction while in the second case one may pick up one of the isotropic directions $x_{i}$ 's for the width of the strip. In what follows we consider both of them.

\section{Case A}

For the first case, where the width of strip is along the anisotropic scaling direction, consider the following strip

$$
\frac{\ell}{2} \leq y \leq \frac{\ell}{2}, \quad 0 \leq x_{i} \leq L, \text { for } i=1, \cdots, d-1 .
$$

Now the aim is to minimize a surface on the bulk whose boundary is the above strip and its profile in the bulk is given by $y=y(r)$. From the geometry (1.7) the induced metric on this surface reads

$$
d s_{i n d}^{2}=r^{2 \frac{\theta}{d}}\left[\left(\frac{y^{\prime 2}}{r^{2 z}}+\frac{1}{r^{2}}\right) d r^{2}+\frac{\sum_{i=1}^{d-1} d x_{i}^{2}}{r^{2}}\right] .
$$

Therefore the area of the surface is

$$
A=L^{d-1} \int_{\epsilon} d r \frac{\sqrt{r^{2(z-1)}+y^{\prime 2}}}{r^{d+z-\theta-1}} .
$$

where prime represents derivative with respect to $r$. Note that since we are considering $\theta \leq d$, one has $d+z-\theta-1 \geq 0$. Therefore the area always diverges at UV where $r \rightarrow 0$, and thus we had to introduce a UV cut off $\epsilon$ in the above expression.

To minimize the area one may consider the above expression as an action of a one dimensional mechanical system whose momentum is a constant of motion

$$
\frac{1}{r^{d+z-\theta-1}} \frac{y^{\prime}}{\sqrt{r^{2(z-1)}+y^{\prime 2}}}=\frac{1}{r_{0}^{d+z-\theta-1}}=\text { constant. }
$$


Here $r_{0}$ is the turning point where $\left.y^{\prime}\right|_{r_{0}} \rightarrow \infty$. The above constraint can be solved to find the width of the strip as a function of turning point $r_{0}$,

$$
l=2 r_{0}^{z} \int_{0}^{1} d \xi \frac{\xi^{d+2 z-\theta-2}}{\sqrt{1-\xi^{2(d+z-\theta-1)}}} .
$$

Finally for the area we arrive at

$$
A=\frac{L^{d-1}}{r_{0}^{d-\theta-1}} \int_{\frac{\epsilon}{r_{0}}}^{1} d \xi \frac{\xi^{\theta-d}}{\sqrt{1-\xi^{2(d+z-\theta-1)}}}
$$

It is now possible to preform the above integrals and eliminate $r_{0}$ between them to find the entanglement entropy as a function of width $\ell$. For $\theta \neq d-1$, one finds

$$
\ell=c_{0} r_{0}^{z}, \quad A=\frac{L^{d-1}}{d-\theta-1}\left(\frac{1}{\epsilon^{d-\theta-1}}-\frac{b_{0}}{r_{0}^{d-\theta-1}}\right)
$$

with

$$
c_{0}=\frac{2 \sqrt{\pi}}{z} \frac{\Gamma\left(\frac{d+2 z-\theta-1}{2(d+z-\theta-1)}\right)}{\Gamma\left(\frac{z}{2(d+z-\theta-1)}\right)}, \quad b_{0}=\sqrt{\pi} \frac{\Gamma\left(\frac{-d+\theta+1}{2(d+z-\theta-1)}\right)}{\Gamma\left(\frac{z}{2(d+z-\theta-1)}\right)} .
$$

Plugging the obtained minimized area in the equation (2.1), one can find the entanglement entropy as follows

$$
S=\frac{L^{d-1}}{4(d-\theta-1) G_{N}}\left(\frac{1}{\epsilon^{d-\theta-1}}-b_{0} \frac{c_{0}^{(d-\theta-1) / z}}{\ell^{(d-\theta-1) / z}}\right) .
$$

As we already mentioned, one would not expect that the metric provides the gravitational dual for whole range of the energies. In fact above a given dynamical scale, $r_{F}$, one would expect that the UV theory will be described by a UV completion geometry $20{ }^{4}$. Taking into account the dynamical scale $r_{F}$, the entanglement entropy reads

$$
S=\frac{L^{d-1}}{4(d-\theta-1) G_{N}}\left[\left(\frac{\epsilon}{r_{F}}\right)^{\theta} \frac{1}{\epsilon^{d-1}}-\left(\frac{\ell^{1 / z}}{r_{F}}\right)^{\theta} \frac{b_{0} c_{0}^{(d-\theta-1) / z}}{\ell^{(d-1) / z}}\right]
$$

On the other hand for $\theta=d-1$ and any $z$, one gets $z \ell=2 r_{0}^{z}$ while for the area one finds $A=-L^{d-1} \ln \frac{\epsilon}{2^{1 / z} r_{0}}$. Therefore in this case, considering the scaling $r_{F}$, one

${ }^{4}$ With the dynamical scale $r_{F}$ the metric 1.3 should be read

$$
d s_{d+2}^{2}=\left(\frac{r}{r_{F}}\right)^{\frac{2 \theta}{d}}\left(-\frac{d t^{2}}{r^{2 z}}+\frac{d r^{2}}{r^{2}}+\frac{\sum_{i=1}^{d} d x_{i}^{2}}{r^{2}}\right)
$$


arrives at

$$
S=\frac{1}{4 z \pi G_{N}} \frac{L^{d-1}}{r_{F}^{d-1}} \ln \frac{z \ell}{\epsilon^{z}}
$$

showing that the dual theory exhibits logarithmic violation for the area law which could be an indicator of having a Fermi surface in the model. Note also that for $d=\theta$, the expression for $A$ is finite and indeed for the entropy we find

$$
S \sim L^{d-1} \ell^{1 / z}
$$

It is interesting to note that, even though in the present case where the anisotropic scaling was along a spatial direction, the system exhibits similar behaviors as that in [20] where the anisotropic scaling was along the time direction. Actually the results are almost identical up to a $1 / z$ factor in the power which can be understood from the fact that the scaling of the width of the strip in these two cases are related by factor of $z$. Therefore from the results of [20] one can find our results by just replacing $r_{0} \rightarrow r_{0}^{z}$, or $\ell \rightarrow \ell^{1 / z}$.

\section{Case B}

Let us consider a case where the width of strip is along one of $x_{i}$ 's directions. The corresponding strip subsystem may be given by

$$
\frac{\ell}{2} \leq x_{d-1} \leq \frac{\ell}{2}, \quad 0 \leq y \leq L, \quad 0 \leq x_{i} \leq L, \quad \text { for } i=1, \cdots, d-2 .
$$

The holographic entanglement entropy is given by a minimal surface whose shape at the boundary coincides to the above stripe and has a profile in the bulk given by $x_{1}=x(r)$. The induced metric on the surface is

$$
d s_{i n d}^{2}=r^{2 \frac{\theta}{d}}\left[\frac{d y^{2}}{r^{z}}+\left(1+x^{2}\right) \frac{d r^{2}}{r^{2}}+\frac{\sum_{i=1}^{d-2} d x_{i}^{2}}{r^{2}}\right]
$$

Thus the area reads

$$
A=L^{d-1} \int d r \frac{\sqrt{1+x^{\prime 2}}}{r^{d+z-\theta-1}}
$$

One can go through the well known procedure to minimize the surface, as we did in the previous case. Doing so, one arrives at the following expressions for the width $\ell$ and area $A$

$$
\ell=2 r_{0} \int_{0}^{1} d \xi \frac{\xi^{d+z-\theta-1}}{\sqrt{1-\xi^{2(d+z-\theta-1)}}}, \quad A=\frac{L^{d-1}}{r^{d+z-\theta-2}} \int_{\frac{\epsilon}{r_{0}}}^{1} d \xi \frac{\xi^{-(d+z-\theta-1)}}{\sqrt{1-\xi^{2(d+z-\theta-1)}}}
$$

Then it is easy to calculate the above integrals to find the width, $\ell$, and area $A$ as functions of turning point $r_{0}$. By eliminating $r_{0}$ between these two functions, we 
will find the holographic entanglement entropy as a function of width $\ell$. In fact for $\theta \neq d+z-2$ one finds

$$
\ell=c_{0} r_{0}, \quad A=\frac{L^{d-1}}{d+z-\theta-2}\left(\frac{1}{\epsilon^{d+z-\theta-2}}-\frac{b_{0}}{r^{d+z-\theta-2}}\right),
$$

where

$$
c_{0}=\sqrt{\pi} \frac{\Gamma\left(\frac{d+z-\theta}{2(d+z-\theta-1)}\right)}{\Gamma\left(\frac{1}{2(d+z-\theta-1)}\right)}, \quad b_{0}=\sqrt{\pi} \frac{\Gamma\left(\frac{d+z-\theta}{2(d+z-\theta-1)}\right)}{\Gamma\left(\frac{1}{2(d+z-\theta-1)}\right)} .
$$

Therefore, taking into account the dynamical scale $r_{F}$, the entanglement entropy is

$$
S=\frac{L^{d-1}}{4(d+z-\theta-2) G_{N}}\left[\left(\frac{\epsilon}{r_{F}}\right)^{\theta} \frac{1}{\epsilon^{d+z-2}}-\left(\frac{l}{r_{F}}\right)^{\theta} \frac{b_{0} c_{0}^{d+z-\theta-2}}{\ell^{d+z-2}}\right] .
$$

On the other hand for $\theta=d+z-2$ one gets $\ell=2 r_{0}$, while the area exhibits a logarithmic behavior, $A=L^{d-1} \ln \frac{2 r_{0}}{\epsilon}$. Thus the entanglement entropy is found to be

$$
S=\frac{1}{4 G_{N}} \frac{L^{d-1}}{r_{F}^{d+z-2}} \ln \frac{\ell}{\epsilon},
$$

that violates the area law. Here we have also restored the dynamical scale $r_{F}$. If we restrict ourselves to the case of $z \geq 2$ we get the above logarithmic violation of area law just for $d=\theta$. Thus, taking into account that we are interested in $\theta \leq d$, the subsystem defined by (2.14), unlike the case of (2.2), can never have an extensive entropy.

However we note that, if we let the dynamical exponent to be one, $z=1$, then the entanglement entropy of the subsystem (2.14) exhibits logarithmic violation of area for $\theta=d-1$. Moreover for $z=1$ one may have $\theta=d+z-1$ which means $\theta=d$. In this case the area turns out to be a constant leading to an extensive entanglement entropy as follows

$$
S \sim L^{d-1} \ell
$$

Of course it is worth mentioning that in this case the metric (1.3) is, actually, flat space (more precisely, it is a metric of upper half space) and it is not clear what the holographic entanglement entropy means!

\section{Open string probe}

In this section we would like to probe the background 1.3 and 1.7 by an open string. As we already mentioned these geometries are expected to provide gravitational dual for theories with hyperscaling violation in a specific range of energies. 
Indeed these geometries may be though of as an effective holographic description of the dual theories with a UV cut off set by the dynamical scale $r_{F}$ over which the geometries may not provide a good description.

Therefore by probing these geometries with an open string one can explore the interaction of an external object in an effective theory exhibiting hyperscaling violation. In particular we will consider a static or moving open string which in turn can be used to find the effective potential between two external point like objects or the drag force the external object may experience.

\subsection{Wilson loop}

The effective potential between external point like objects may be obtained by computing the Wilson loop in the model. Actually AdS/CFT correspondence has provided a simple prescription to compute the Wilson loop in a strongly coupled field theory by making use of its gravitational dual [27, 28]. In this context the Wilson loop can be computed by minimizing a world sheet of an open string whose ends on the boundary are, indeed, representing the external objects.

To proceed consider the following ansatz for the open string

$$
t=\tau, \quad r=\sigma, \quad x_{1}=x(r),
$$

one the background (1.3). For this ansatz the Nambu-Goto action reads

$$
I=-\frac{1}{2 \pi \alpha^{\prime}} \int d t d r r^{-\frac{d(z+1)-2 \theta}{d}} \sqrt{1+x^{\prime 2}} .
$$

This action can be thought of as a one dimensional mechanical system for $x$ whose momentum is a constant of motion. With this interpretation, denoting the turning point by $r_{0}$ where $x^{\prime} \rightarrow \infty$, one finds

$$
\ell=2 \int_{0}^{r_{0}} d r \frac{\left(\frac{r}{r_{0}}\right)^{\frac{d(z+1)-2 \theta}{d}}}{\sqrt{1-\left(\frac{r^{2}}{r_{0}^{2}}\right)^{\frac{d(z+1)-2 \theta}{d}}}}
$$

where $\ell$ is the distance between two external objects. Note that in the cases we are interested in one has $d(z+1)-2 \theta \geq 0$. It is, then, easy to perform the integral yielding to

$$
\ell=c_{0} r_{0}, \quad \text { with } c_{0}=\sqrt{\pi} \frac{\Gamma\left(\frac{d(2+z)-2 \theta}{2(d(z+1)-2 \theta)}\right)}{\Gamma\left(\frac{d}{2(d(z+1)-2 \theta)}\right)}
$$


On the other hand the on shell action of the string reads

$$
I=-\frac{T}{2 \pi \alpha^{\prime}} \int_{\epsilon}^{r_{0}} d r \frac{r^{-\frac{d(z+1)-2 \theta}{d}}}{\sqrt{1-\left(\frac{r^{2}}{r_{0}^{2}}\right)^{\frac{d(z+1)-2 \theta}{d}}}}=-\frac{T d}{2 \pi(z d-2 \theta) \alpha^{\prime}}\left(\frac{1}{\epsilon^{z-\frac{2 \theta}{d}}}+\frac{I_{0}}{r_{0}^{z-\frac{2 \theta}{d}}}\right)
$$

where $T$ is the time interval over which the Wilson loop is calculated, and

$$
I_{0}=\frac{\sqrt{\pi}}{1+2 z-4 \frac{\theta}{d}} \frac{\Gamma\left(\frac{-d z+2 \theta}{2(d(z+1)-2 \theta)}\right)}{\Gamma\left(\frac{-d(1+2 z)+4 \theta}{2(d(z+1)-2 \theta)}\right)}
$$

Therefore for $z d \neq 2 \theta$, restoring the dynamical scale $r_{F}$, the effective potential reads

$$
V_{\mathrm{eff}}=-\frac{d}{2 \pi(z d-2 \theta) \alpha^{\prime}}\left[\left(\frac{\epsilon}{r_{F}}\right)^{\frac{2 \theta}{d}} \frac{1}{\epsilon^{z}}+\left(\frac{\ell}{r_{F}}\right)^{\frac{2 \theta}{d}} \frac{I_{0} c_{0}^{z-\frac{2 \theta}{d}}}{\ell^{z}}\right] .
$$

While for $z d=2 \theta$ one has $\ell=2 r_{0}$ and for the action one gets

$$
I=-\frac{T}{2 \pi \alpha^{\prime}} \int_{\frac{\epsilon}{r_{0}}}^{1} \frac{d \xi}{\xi \sqrt{1-\xi^{2}}}=-\frac{T}{2 \pi \alpha^{\prime}} \ln \frac{2 r_{0}}{\epsilon} .
$$

Thus in this case the effective potential has a logarithmic behavior as follows

$$
V_{\mathrm{eff}}=-\frac{1}{2 \pi r_{F}^{z} \alpha^{\prime}} \ln \frac{\ell}{\epsilon}
$$

So far we have considered an open string probing the metric 11.3 . We would like to extend our study for the metric (1.7) too. In this case we recognize two distinctive cases depending on whether the distance between the external objects is along the anisotropic scaling direction, $y$, or along one of $x_{i}$ 's directions. Actually for both of cases one may go through the similar computations as presented above to calculate the effective potential between two point like external objects. Doing so one arrives at

$$
\begin{array}{r}
\text { Along } y \text { direction } \quad V_{\text {eff }} \sim \begin{cases}-\ell^{\frac{2 \theta-d}{z d}} & \theta \neq \frac{d}{2}, \\
-\frac{1}{z} \ln \frac{\ell}{\epsilon} & \theta=\frac{d}{2},\end{cases} \\
\text { Along } x_{1} \text { direction } \quad V_{\text {eff }} \sim \begin{cases}-\ell^{\frac{2 \theta-d}{d}} & \theta \neq \frac{d}{2}, \\
-\ln \frac{\ell}{\epsilon} & \theta=\frac{d}{2} .\end{cases}
\end{array}
$$

It is worth to note that since $\theta \leq d$, it is possible (for example for $\theta=d$ ) to get linear or fractional power law effective potential. In fact due to the anisotropic in 
the spatial directions, it is possible to get a power law effective potential in one direction while a confining potential in other directions.

\subsection{Drag force}

Let us consider an open string moving in the background 1.3 which could represent an external point like moving source in an effective theory with hyperscaling violation. This can be used to study the drag force that the external object might feel $[29]^{5}$. Drag force for non-relativistic theories with Schrödinger or Lifshitz symmetries have been studied in [31] and [32], respectively, where it was shown that even at zero temperature the drag force is non-zero. It is indeed the aim of this subsection to study the drag force for theories with hyperscaling violation.

Consider the following ansatz for the moving string

$$
t=\tau, \quad r=\sigma, \quad x_{1}=v t+x(r),
$$

and all other coordinates are set to zero. Then the Nambu-Goto action for this string reads

$$
I=-\frac{1}{2 \pi \alpha^{\prime}} \int d t d r r^{2 \frac{\theta}{d}-2} \sqrt{r^{2(1-z)}\left(1+x^{\prime 2}\right)-v^{2}},
$$

Since the metric components are $t$ independent, the above action may be treated as a one dimensional mechanical system whose momentum is the constant of motion

$$
r^{2 \frac{\theta}{d}-2} \frac{r^{2(1-z)} x^{\prime}}{\sqrt{r^{2(1-z)}\left(1+x^{\prime 2}\right)-v^{2}}}=-2 \pi \alpha^{\prime} \Pi_{x}=\text { constant }
$$

which can be solved to find $x^{\prime}$ as follows

$$
x^{\prime 2}=4 \pi^{2} \alpha^{\prime 2} \Pi_{x}^{2} \frac{1-r^{2(z-1)} v^{2}}{r^{4 \frac{\theta}{d}-2(z+1)}-4 \pi \alpha^{2} \Pi_{x}^{2}} .
$$

In terms of the constant $\Pi_{x}$, energy, $E$, and momentum, $P$, that the open string gains from through its end point are given by

$$
\frac{d E}{d t}=\Pi_{x} v . \quad \frac{d P}{d t}=\Pi_{x} .
$$

The constant $\Pi_{x}$ can be obtained by requiring that the numerator and denominator of (3.14) vanish at the same point, imposed by the fact that $x^{\prime}$ should be real. Setting the numerator to zero one finds $v=r_{0}^{1-z}$. From this solution we observe that as we are varying $r_{0}$ from infinity to zero (flowing from IR to UV), the velocity takes its value from zero to infinity. This is, in fact, due to the non-relativistic property

\footnotetext{
${ }^{5}$ After we submitted our paper we were aware that the drag force in the background (1.3) has also been, recently, sudied in 30 .
} 
of the dual field theory. Moreover by plugging the solution $r_{0}$ into the denominator one arrives at

$$
\Pi_{x}=-\frac{v}{2 \pi \alpha^{\prime}} v^{\frac{2(d-\theta)}{d(z-1)}} .
$$

Now consider a single non-relativistic particle with momentum $P$ and mass $M$, then we have $P=M v$. It is useful to formally rewrite the above expression for the drag force in terms of $P$. Then we can perform the integral which for $d \neq \theta$ yielding to

$$
P=\left(P_{0}^{-\frac{2(d-\theta)}{d(z-1)}}+\frac{d-\theta}{d(z-1) \pi \alpha^{\prime}} \frac{t}{M^{\frac{d(z-3)+2 \theta)}{d(z-1)}}}\right)^{-\frac{d(z-1)}{2(d-\theta)}}
$$

while for $d=\theta$ one finds

$$
P=P_{0} e^{-\frac{t}{2 \pi \alpha^{\prime} M}}
$$

Here $P_{0}$ is the initial momentum.

We note that even though the theory we considered here was at zero temperature the drag force is non-zero. In other words, as the open string moves on the metric (1.3) it experiences a non-zero friction. Therefore if we let the open string (external point like object) moves with an initial velocity (initial momentum $p_{0}$ ) after some time it will stop unless one compensates the losing energies by an external force.

Actually having a non-zero drag force even at zero temperature appears when there is an anisotropic scaling between time and the direction the string moves. This is of course a typical feature of non-relativistic field theories whose gravitational duals have metrics with an anisotropic scaling.

In fact it is straightforward to compute the induced metric on the string worldsheet and argue that there is an event. For example for an open string moving in the geometry (1.3) the induced metric on its worldsheet is

$$
d s^{2}=r^{\frac{2 \theta}{2}-2}\left[-\left(r^{2(1-z)}-v^{2}\right) d t^{2}+\left(1+x^{\prime 2}\right) d r^{2}+2 v x^{\prime} d r d t\right],
$$

which can be diagonalized by the following change of coordinates [33

$$
t=\tau+\eta(r), \quad \text { with } \eta^{\prime}(r)=\frac{v x^{\prime}}{r^{2(1-z)}-v^{2}},
$$

by which the induced metric reads

$$
d s^{2}=r^{\frac{2 \theta}{2}-2}\left[-\left(r^{2(1-z)}-v^{2}\right) d \tau^{2}+\frac{r^{\frac{4 \theta}{d}-2(z+1)}}{r^{\frac{4 \theta}{d}-2(z+1)}-4 \pi^{2} \alpha^{2} \Pi_{x}^{2}} d r^{2}\right] .
$$

As we see the induced metric develops an event horizon whose location is given by 
$r_{0}^{2(1-z)}-v^{2}=0$, and the Hawking temperature is

$$
T=\frac{\sqrt{(z-1)\left(z+1-\frac{2 \theta}{d}\right)}}{2 \pi r_{0}^{z}} .
$$

On observes that even the bulk is at zero temperature, the worldsheet is thermal which should be responsible of having energy loss in this case. Note that for $z=1$ the temperature is zero and indeed for this the energy loss is zero too.

Let us now study an open string moving in the metric (1.7). In this case we could consider a situation where either the string moves in $x_{1}$ or $y$ directions. In the first case, actually, due to the rotational symmetry in $x_{1}$ and $t$ directions when the string moves in $x_{1}$ direction, one gets nothing new, except that of the pure AdS case [29]. Namely there is no drag force.

On the other hand when the open string moves in $y$ direction, the induced metric on its worldvolume is

$$
d s^{2}=r^{\frac{2 \theta}{2}-2}\left[-\left(1-v^{2} r^{2(1-z)}\right) d t^{2}+\left(1+x^{\prime 2} r^{2(1-z)}\right) d r^{2}+2 v x^{\prime} r^{2(1-z)} d r d t\right],
$$

which, by making use of the equation of motion of $\phi$, can be recast to the following form

$$
d s^{2}=r^{\frac{2 \theta}{2}-2}\left[-\left(1-v^{2} r^{2(1-z)}\right) d \tau^{2}+\frac{r^{\frac{4 \theta}{d}-2(z+1)}}{r^{\frac{4 \theta}{d}-2(z+1)}-4 \pi^{2} \alpha^{2} \Pi_{x}^{2}} d r^{2}\right]
$$

It seems that the resultant world-volume metric has an event horizon and the situation could be the same as before. We note, however, that if we went through the standard procedure, as we did in the previous case, the Hawking temperature turns out to be imaginary which could mean that the ansatz we considered for the open string is not a consistent solution. Therefore it seems that even in this case where the string moves along a direction with an anisotrpoic scaling, the drag force is zero.

\subsection{Rotating open string}

As a final example of open strings probing the geometries (1.3) and (1.7), in this subsection we will consider a rotating open string in the bulk that describes, holographically, an external point like object in the strongly coupled theories with hyperscaling violation undergoes circular motion. Such a study has been first done for a rotating quark in strongly coupled $\mathcal{N}=4$ SYM theory in 34, 35] where the authors "have computed the energy density and angular distribution of the power radiated by a rotating quark in this theory." 6 To start let us consider the following

\footnotetext{
${ }^{6}$ Rotating massive quark in an anisotropic strongly coupled plasma has also been studied [36. For rotating strings on non-conformal holography see 37 .
} 
change of coordinates in the metrics $(1.3)$ and 1.7$)$

$$
\left(x_{1}, x_{2}\right) \rightarrow(\rho, \phi) ; \quad \text { such that } d x_{1}^{2}+d x_{2}^{2}=d \rho^{2}+\rho^{2} d \phi^{2},
$$

by which the metrics (1.3) and 1.7) may be recast to the following forms

$$
\begin{aligned}
& d s_{d+2}^{2}=r^{\frac{2 \theta}{d}}\left(-\frac{d t^{2}}{r^{2 z}}+\frac{d r^{2}}{r^{2}}+\frac{d \rho^{2}}{r^{2}}+\frac{\rho^{2} d \phi^{2}}{r^{2}}+\frac{\sum_{i=3}^{d} d x_{i}^{2}}{r^{2}}\right) \\
& d s_{d+2}^{2}=r^{\frac{2 \theta}{d}}\left(\frac{d y^{2}}{r^{2 z}}+\frac{d r^{2}}{r^{2}}+\frac{d \rho^{2}}{r^{2}}+\frac{\rho^{2} d \phi^{2}}{r^{2}}+\frac{\sum_{i=3}^{d-1} d x_{i}^{2}}{r^{2}}-\frac{d t^{2}}{r^{2}}\right) .
\end{aligned}
$$

Let us now consider the following ansatz for a rotating open string in these backgrounds

$$
t=\tau, \quad r=\sigma, \quad \rho=\rho(r), \quad \phi=\omega t+\phi(r),
$$

and all other coordinates are set to zero. From dual field theory point of view this corresponds to an extremal point like object undergoes a circular motion around the center $\rho=0$ with frequency $\omega$.

The Nambu-Goto action for this open string in the background (3.26) is

$$
I=-\frac{1}{2 \pi \alpha^{\prime}} \int d t d r r^{2 \frac{\theta}{d}-1-z} \sqrt{\left(1-\rho^{2} \omega^{2} r^{2(z-1)}\right)\left(1+\rho^{\prime 2}\right)+\rho^{2}{\phi^{\prime}}^{2}},
$$

whereas for the metric (3.27) it becomes

$$
I=-\frac{1}{2 \pi \alpha^{\prime}} \int d t d r r^{2 \frac{\theta}{d}-2} \sqrt{\left(1-\rho^{2} \omega^{2}\right)\left(1+{\rho^{\prime}}^{2}\right)+\rho^{2}{\phi^{\prime}}^{2}} .
$$

Note that the action (3.30) up to a factor of $r^{2 \frac{\theta}{d}}$ is the same as that for pure AdS case studied in [35] .

In both cases the actions are independent of $\phi(r)$, and therefore the corresponding angular momentum, $\Pi_{\phi}=-\frac{\partial \mathcal{L}}{\partial \phi^{\prime}}$, is an integral of motion. In other words for the action (3.29), setting $p=2 \frac{\theta}{d}-1-z$ and $q=2(z-1)$ one has

$$
\Pi_{\phi}=\frac{-r^{p} \rho^{2} \phi^{\prime}}{\sqrt{\left(1-\rho^{2} \omega^{2} r^{q}\right)\left(1+\rho^{\prime 2}\right)+\rho^{2}{\phi^{\prime}}^{2}}}=\text { constant }
$$

while for action (3.30), setting $\tilde{p}=2 \frac{\theta}{d}-2$ one gets

$$
\Pi_{\phi}=\frac{-r^{\tilde{p}} \rho^{2} \phi^{\prime}}{\sqrt{\left(1-\rho^{2} \omega^{2}\right)\left(1+\rho^{\prime 2}\right)+\rho^{2} \phi^{\prime 2}}}=\text { constant. }
$$


Using these expressions one can find the equation of motion for $\phi(r)$ as follows

$$
\begin{aligned}
& \text { for action } \quad \phi^{\prime 2}=\frac{\Pi_{\phi}^{2}\left(1-\omega^{2} \rho^{2} r^{q}\right)\left(1+\rho^{\prime 2}\right)}{\rho^{2}\left(r^{2 p} \rho^{2}-\Pi_{\phi}^{2}\right)}, \\
& \text { for action } 3.30) \quad \phi^{\prime 2}=\frac{\Pi_{\phi}^{2}\left(1-\omega^{2} \rho^{2}\right)\left(1+\rho^{\prime 2}\right)}{\rho^{2}\left(r^{2} \tilde{p} \rho^{2}-\Pi_{\phi}^{2}\right)} .
\end{aligned}
$$

Finally, utilizing the above expressions, the equation of motion for $\rho(r)$, is then given by

$$
\begin{aligned}
& \text { for action }(3.29) \quad \rho^{\prime \prime}+\left(\frac{\rho\left(r-p \rho \rho^{\prime}\right)}{r\left(r^{-2 p} \prod_{\phi}^{2}-\rho^{2}\right)}+\frac{\left(2-q r^{q-1} \omega^{2} \rho^{3} \rho^{\prime}\right)}{2 \rho\left(1-\rho^{2} \omega^{2} r^{q}\right)}\right)\left(1+\rho^{2}\right)=0 \text {, } \\
& \text { for action } \quad \rho^{\prime \prime}+\left(\frac{\rho(r-30)}{r\left(r^{-2 \tilde{p}} \Pi_{\phi}^{2}-\rho^{\prime}\right)}+\frac{1}{\rho\left(1-\rho^{2} \omega^{2}\right)}\right)\left(1+\rho^{\prime 2}\right)=0 .
\end{aligned}
$$

We have now all equations to study the rotating strings. To proceed, in what follows we will only consider the first case which is based on the action $(3.29)$. Actually the results for the second case can be obtained from the first one by replacing $p \rightarrow \tilde{p}$ and $q \rightarrow 0$.

In general to find a solution for the rotating string we have to solve the equations of motion of $\rho$ and $\phi$ with a given initial data 35]. Actually to solve the equation of motion for $\rho$ we find that it is singular when $\Pi_{\phi}^{2}-\rho^{2} r^{2 p}=0$ or $1-\rho^{2} \omega^{2} r^{q}=0$. On the other hand in order to impose the reality condition on $\phi^{\prime}$ one has to assume that these two expressions should vanish at the same point. Let us denote by $r_{0}$ the point where both of the expressions vanish. Then one gets

$$
\rho_{0}=\frac{\left(\Pi_{\phi} \omega\right)^{\frac{-q}{2 p-q}}}{\omega}, \quad r_{0}=\left(\Pi_{\phi} \omega\right)^{\frac{2}{2 p-q}},
$$

where $\rho_{0}=\rho\left(r_{0}\right)$. On the other hand $\rho^{\prime}\left(r_{0}\right)$ may also be fixed by $\omega$ and $\Pi_{\phi}$ as follows. Following [35 let us expand $\rho$ around $r_{0}$,

$$
\rho=\rho_{0}+\rho_{1}\left(r-r_{0}\right)+\frac{1}{2} \rho_{2}\left(r-r_{0}\right)^{2}+\cdots .
$$

Plugging this expansion in the equation of motion for $\rho$ and requiring all terms in the expansion vanish identically we can read the coefficients $\rho_{i}$. At the first order, for $2 p+q \neq 0$, one finds

$$
\rho_{1}^{2}-\frac{2\left(2 \omega^{2}-p q\left(\Pi_{\phi} \omega\right)^{\frac{2 q+4}{q-2 p}}\right)}{(q+2 p) \omega\left(\Pi_{\phi} \omega\right)^{\frac{q+2}{q-2 p}}} \rho_{1}-1=0,
$$

which can be solved to find $\rho_{1}$ in terms of $\omega$ and $\Pi_{\phi}$. 


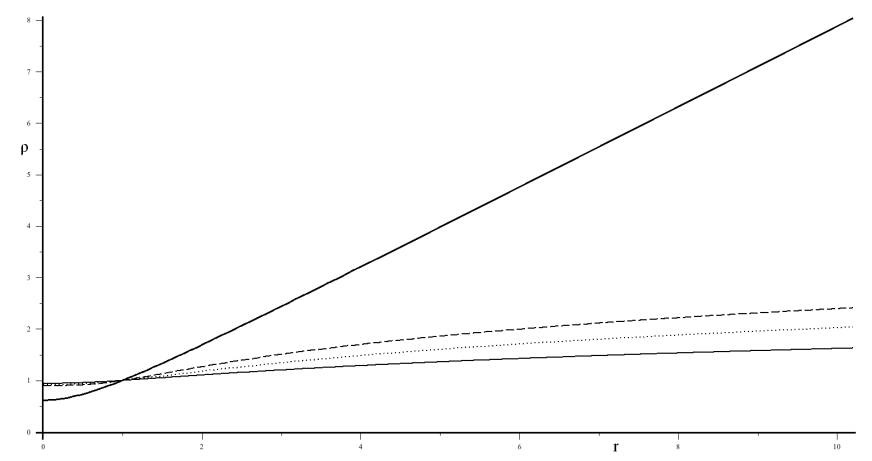

Figure 1: $\rho$ as a function of $r$ for the cases of $z=2$ and $\theta=\frac{3 d}{4}, \frac{d}{2}, 0$ which are shown by dashed, dotted and thin curves, respectively. In order to compare our results with that of AdS case $(z=1, \theta=0)$, we have also plotted this case which is shown by a thick curve. Note that to solve the equation we have set the initial date as $\omega=\Pi_{\phi}=1$.

Therefore with $\omega$ and $\Pi_{\phi}$ as the initial data one should be able to find a unique solution for the rotating open string. Indeed, by making use of a numerical method we have solved the equation of motion for $\rho$ with specific values of $p$ and $q$. In particular in the figure (1) we have plotted $\rho$ as a function of $r$ for the cases of $q=2$ and $p=-3 / 2,-2,-3$ which correspond to the cases where $z=2$ and $\theta=\frac{3 d}{4}, \frac{d}{2}, 0$. In order to compare the results with that in the pure AdS case [35], we have also plotted $\rho$ as a function of $r$ for the case of $z=1, \theta=0$.

The function of $\rho(r)$ shows how the string spreads as a function depth [35]. Actually as we can see from the figure (1) for the rotating string in the AdS case, the string spreads linearly, $\rho \sim r$, as we approach the IR region (see also the exact solution given in [35]). On the other hand although in the present case the string still spreads as a function of depth, the rate of spreading is slower than that in the AdS case. In fact in our case, as one can see from our numerical solution, the asymptotic behavior is $\rho \sim r^{\alpha}$ for $r \gg 1$ with $\alpha<1$. Of course for $r<1$ the string on the hyperscaling violation metrics spreads faster than that on the pure AdS (see figure 1). Pictorially, these behaviors can be visualized by projecting the function $\rho(\phi)$ to a $r=$ constant (boundary) surface as we have depicted in figure (2).

On the other hand looking at the behavior of the function $\phi$ which describes how the string winds in azimuthal angle as a function of depth [35], one finds that in the present case where we have the hyperscaling violation, it grows faster that that in AdS as we move toward the core of the space-time.

For $2 p+q=0$, expanding $\rho$ around $\rho_{0}$ we find that all $\rho_{i}=0$. Indeed it is easy to show that in this case $\rho=$ constant is an exact solution of the equation of motion where the constant is found to be $\rho=\sqrt{\Pi_{\phi} / \omega}$ which is consistent with (3.36). Moreover, in this case the equation of motion of $\phi$ can also be solved exactly. 

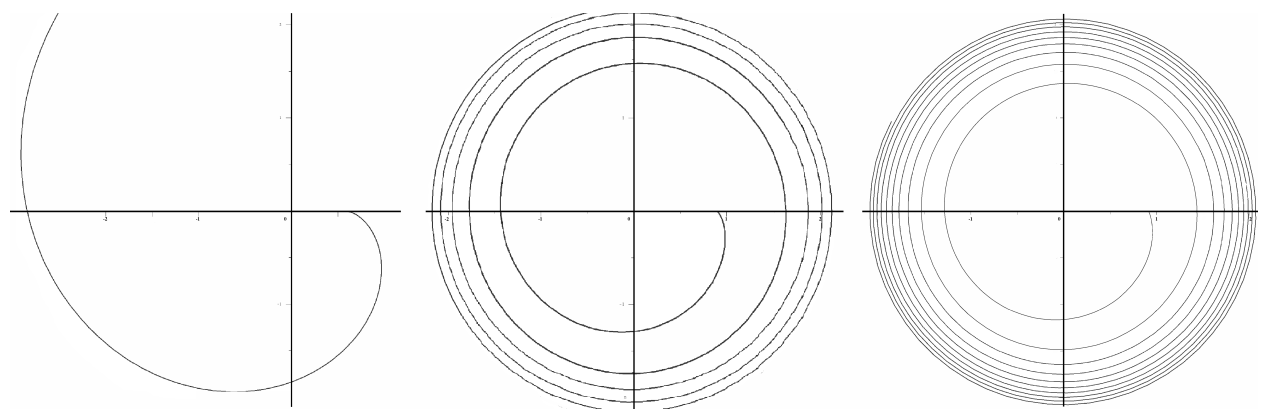

Figure 2: Here we have plotted $\rho$ as a function of $\phi$ for the cases of $z=1, \theta=0$ (left), $z=2, \theta=0$ (middle) and $z=2, \theta=\frac{d}{2}$ (right). The function is projected in to $x_{1}-x_{2}$ plane, where $\rho$ and $\phi$ are polar coordinates of this place. Here we have set $\omega=\Pi_{\phi}=1$.

In fact for $2 p+q=0$, which corresponds to $\theta=d$ for all values of $z$, one finds 7

$$
\phi=\omega\left(t+\frac{r^{z}}{z}\right), \quad \rho=\rho_{0}=\sqrt{\frac{\Pi_{\phi}}{\omega}}, \quad r_{0}=\left(\Pi_{\phi} \omega\right)^{\frac{1}{2(1-z)}} .
$$

To wrap up this section we note that looking at the induced metric of the string worldsheet one observes that it has an even horizon. More precisely, with a proper change of coordinates, the induced metric of the string worldsheet is found

$$
d s^{2}=r^{\frac{2 \theta}{d}-2-q}\left[-\left(1-\omega^{2} \rho^{2} r^{q}\right) d \tau^{2}+\frac{r^{2 p+q} \rho^{2}\left(1+\rho^{\prime 2}\right)}{r^{2 p} \rho^{2}-\Pi_{\phi}^{2}} d r^{2}\right],
$$

which has an even horizon at $r=r_{0}$. The corresponding Hawking temperature is

$$
T=\frac{1}{4 \pi \rho_{0} r_{0}^{z}} \sqrt{-\frac{2\left(q \rho_{0}+2 \rho_{1} r 0\right)\left(p \rho_{0}+\rho_{1} r_{0}\right)}{1+\rho_{1}^{2}}} .
$$

It is crucial to note that to have a solution for $\rho(r)$ in the vicinity of $r_{0}$, the positivity condition of the right hand side of the equation (3.33) reduces to the following inequality

$$
\left(q \rho_{0}+2 \rho_{1} r 0\right)\left(p \rho_{0}+\rho_{1} r_{0}\right)<0,
$$

which is necessary to have a well defined real Hawking temperature.

Having found the shape of the rotating string, it is then possible to compute the radiation power of the string. From the Nambu-Goto action one can obtain the energy density and energy flux of string from which the energy loss rate of the string

\footnotetext{
${ }^{7}$ Note that this solution is not valid for $z=1$. Actually for $\theta=d$ and $z=1$ the geometry reduces to the flat space-time where we do not have a well defined holography.
} 


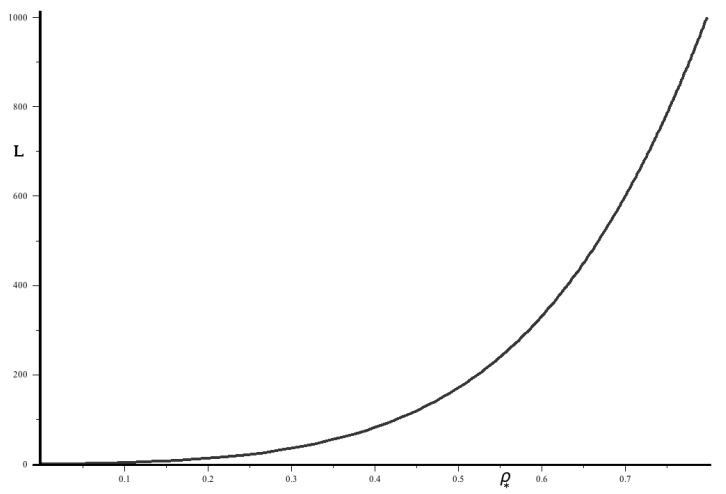

Figure 3: Here we have plotted $\rho_{*}$ as a function of $L=\Pi_{\phi} \omega$ for the case of $z=$ $2, \theta=0$. Essentially this is the energy loss rate in terms of the radius of circle the external object moves.

is found 35

$$
\frac{d E}{d t}=\frac{\Pi_{\phi} \omega}{2 \pi \alpha^{\prime}}
$$

Of course the goal is to compute the energy loss rate in terms of the parameters of the dual field theory which is frequency and the radius of circle the external object moves around, i.e. $\rho_{*}=\rho(r \rightarrow 0)$. With the numerical solution we have obtained, it is easy to find $L=\Pi_{\phi} \omega$ as a function of $\rho_{*}$ which is indeed the energy loss rate up to factor of $1 / 2 \pi \alpha^{\prime}$. In the figure (3) we have drown the function of $L$ in terms of $\rho_{*}$ for the case of $z=2, \theta=0$. Form our numerical solution, for large $\rho_{*}$, one finds $L \sim \rho_{*}^{4}$.

As a final remark we note that $r_{0}$ fixes the local velocity of string. Since $r_{0}$ can change from zero to infinity, it means that the local velocity could be infinity, indicating the non-relativistic nature of the dual theory.

\section{Closed string probe}

Since it is possible to find a geometry with non-trivial hyperscaling exponent from string theory (see for example $[20,38,39]$ ) it is natural to study a closed string propagating in this background which could be dual to an operator in the dual field theory with $\mathrm{n}$ hyperscaling violation. In fact in this section, for completeness of our study, we will probe the geometry (1.3) by a closed string. Via AdS/CFT correspondence a closed string moving in the bulk should correspond to a field (operator) configuration with specific energy and probably some other quantum numbers. Therefore it could be possible to study certain properties of the dual theory by making use of a semi-classical closed string on the bulk. In what follows we will consider a folded rotating closed [40] and circular pulsating [41] strings in 
the background (1.3).8

\subsection{Folded closed string}

In this subsection we study a solution representing a rotating closed string configuration which is stretched along the radial coordinate. In order to study this system one needs to write an action for this closed string. Let us parameterize the string worldsheet by $\sigma$ and $\tau$. We can fix the reparameterization invariance by a parameterization such that the time coordinate of space-time, $t$ to be equal to worldsheet time, i.e. $t=\tau$. In this gauge a closed string configuration representing a rotating string with angular velocity $\omega$ on geometry $(1.3)$ stretched along the radial coordinate is given by

$$
\begin{aligned}
& t=\tau, \quad \phi_{d-1}=\phi=\omega \tau, \quad r(\sigma)=r(\sigma+2 \pi), \\
& \phi_{i}=\frac{\pi}{2}(\text { for } i=1 \cdots d-2), \quad \rho=\rho_{0}=\text { constant }
\end{aligned}
$$

where $\sigma$ and $\tau$ are coordinates of the string world sheet. Moreover we parametrize the $d$-dimensional Euclidean space in the metric (1.3) as follows

$$
\sum_{i=1}^{d} d x_{i}^{2}=d \rho^{2}+\rho^{2}\left(d \phi_{1}^{2}+\sin ^{2} \phi_{1} d \phi_{2}^{2}+\cdots+\sin ^{2} \phi_{1} \ldots \sin ^{2} \phi_{d-2} d \phi_{d-1}^{2}\right) .
$$

For this ansatz the Nambu-Goto action, reads

$$
I=-4 \frac{T}{2 \pi \alpha^{\prime}} \int_{0}^{r_{0}} r^{\frac{2 \theta}{d}-2} \sqrt{r^{2-2 z} \dot{t}^{2}-\rho_{0}^{2} \dot{\phi}^{2}} d r
$$

where $d o t$ represents derivative with respect to $\tau$ and $r_{0}=\left(\rho_{0} \omega\right)^{1 /(1-z)}$. The factor of 4 comes from the fact that we are dealing with a folded closed string. Working with one fold string, the string can be divided to four segments. Using the periodicity condition we just need to perform the integral for one quarter of string multiplied by factor 4 . When the periodicity condition is satisfied, using the above Nambu-Goto action the conserved quantities are given by

$$
E=\frac{2 T}{\pi \alpha^{\prime}} \int_{0}^{r_{0}} d r \frac{r^{\frac{2 \theta}{d}-2 z}}{\sqrt{r^{2-2 z}-\rho_{0}^{2} \omega^{2}}}, \quad S=\frac{2 T \rho_{0}^{2} \omega}{\pi \alpha^{\prime}} \int_{0}^{r_{0}} d r \frac{r^{\frac{2 \theta}{d}-2}}{\sqrt{r^{2-2 z}-\rho_{0}^{2} \omega^{2}}},
$$

From these expressions we could find a relation between $E$ and $S$. Actually the above integrals can be performed exactly, though generically they diverge and need

\footnotetext{
${ }^{8}$ Circular pulsating closed string on the confining geometry has also been studied in 42 .
} 
to be regularized. Indeed setting $x=r / r_{0}$, one has

$$
\begin{aligned}
& \mathcal{E}=\frac{\pi \alpha^{\prime}}{2 T} E=r_{0}^{\frac{2 \theta}{d}-z} \int_{0}^{1} \frac{x^{\frac{2 \theta}{d}-z-1}}{\sqrt{1-x^{2 z-2}}} d x=\mathcal{E}_{0} r_{0}^{\frac{2 \theta}{d}-z} \\
& \mathcal{S}=\frac{\pi \alpha^{\prime}}{2 T \rho_{0}} S=r_{0}^{\frac{2 \theta}{d}-1} \int_{0}^{1} \frac{x^{\frac{2 \theta}{d}+z-3}}{\sqrt{1-x^{2 z-2}}} d x=\mathcal{S}_{0} r_{0}^{\frac{2 \theta}{d}-1}
\end{aligned}
$$

where constants $\mathcal{E}_{0}$ and $\mathcal{S}_{0}$ are given by

$$
\mathcal{E}_{0}=\frac{\sqrt{\pi}}{2(z-1)} \frac{\Gamma\left(\frac{2 \theta-z d}{2 d(z-1)}\right)}{\Gamma\left(\frac{2 \theta-1}{2 d(z-1)}\right)}, \quad \mathcal{S}_{0}=\frac{\sqrt{\pi}}{2(z-1)} \frac{\Gamma\left(\frac{2 \theta+z d-2 d}{2 d(z-1)}\right)}{\Gamma\left(\frac{2 \theta+2 z d-3 d}{2 d(z-1)}\right)} .
$$

Therefore with a proper regularization one finds

$$
\mathcal{E}=\mathcal{E}_{0} \mathcal{S}_{0}^{\frac{z d-2 \theta}{2 \theta-d}} \mathcal{S}^{\frac{2 \theta-z d}{2 \theta-d}}
$$

Looking at the explicit form of the energy and spin, depending on the nature of the divergences we recognize two distinct cases. Actually although in a typical case we get power law divergence, there are cases where we get logarithmic divergences that correspond to the cases where either $2 \theta=z d$, or $\theta=0, z=2$. Note that the latter case is indeed Lifshitz geometry with scaling exponent $z=2$.

For $2 \theta=z d$ the energy diverges due to UV divergence of the integral. Therefore we need to regularize it by performing the integral with a UV cut off $\epsilon$. Doing so, one arrives at

$$
\mathcal{E}=\int_{\epsilon}^{r_{0}} \frac{d r}{r \sqrt{1-\left(\frac{r}{r_{0}}\right)^{2(z-1)}}}=\ln \frac{r_{0}}{\epsilon}+\frac{1}{z-1} \ln 2 .
$$

On the other hand in this case one has $\mathcal{S}=\frac{r^{z-1}}{z-1}$. Therefore one finds

$$
\mathcal{E}=\frac{1}{z-1} \ln \frac{2(z-1) \mathcal{S}}{\epsilon^{z-1}}
$$

In the case of $\theta=0, z=2$, although the energy and spin both diverge logarithmically, the relation between energy and spin is still given by the equation (4.8) for $\theta=0$ and $z=2$.

It is also obvious from the equation (4.8) that the expression becomes ill-defined for $\theta=\frac{d}{2}$. In fact in this case starting from the original expression for the energy and spin in the equation 4.5 one finds that the spin is a constant for any turning point, i.e. $\mathcal{S}=\frac{\pi}{2(z-1)}$, while the energy is zero.

It is interesting to note that although the closed string, depending on $\omega$ could be short or long, the relation between energy and spin remains unchanged in all cases discussed above. It is in contrast to the AdS case where for short string $(\omega \rightarrow \infty)$ we find the flat space Regge trajectory, while for long string $(\omega \rightarrow 0)$ the energy in 
terms of spin has logarithmic behavior [40].

\subsection{Circular pulsating string}

In this subsection we will consider a pulsating string which wrapped $m$ times around $\phi_{d-1}$ direction. In other words we study a circular string that expand and contract in the radial direction. The corresponding ansatz for the circular pulsating string is

$$
t=\tau, \quad r=r(t), \quad \phi_{d-1}=m \sigma, \quad \phi_{i}=\frac{\pi}{2}, \quad \rho=\rho_{0}=\text { constant }
$$

For this configuration the string action reads

$$
I=-\frac{m \rho_{0}}{\alpha^{\prime}} \int d t r^{2 \frac{\theta}{d}-z-1} \sqrt{1-r^{2(z-1)} \dot{r}^{2}}
$$

Setting $\xi=r^{z} / z$ the above action can be recast to the following form

$$
I=-\frac{m \rho_{0}}{\alpha^{\prime}} \int d t f(\xi) \sqrt{1-\dot{\xi}^{2}}, \quad \text { with } f(\xi)=(z \xi)^{\frac{2 \theta-z d-d}{z d}} .
$$

This action can be treated as a one-dimensional quantum mechanical system whose Hamiltonian is

$$
H=\sqrt{\Pi^{2}+\left(\frac{m \rho_{0}}{\alpha^{\prime}}\right)^{2} f^{2}(\xi)}
$$

where $\Pi$ is the canonical momentum given by

$$
\Pi=\frac{m \rho_{0}}{\alpha^{\prime}} f(\xi) \frac{\dot{\xi}}{\sqrt{1-\dot{\xi}^{2}}} .
$$

Following [41] $H^{2}$ can be considered as a one dimensional quantum mechanical system with the potential $\left(\frac{m \rho_{0}}{\alpha^{\prime}}\right)^{2} f^{2}(\xi)$. Therefore using the Bohr-Sommerfeld quantization we have

$$
\left(n+\frac{1}{2}\right) \pi=E \int_{\xi_{0}}^{\infty} d \xi \sqrt{1-\left(\frac{f(\xi)}{f\left(\xi_{0}\right)}\right)^{2}},
$$

where $\xi_{0}$ defined by the root of $f\left(\xi_{0}\right)=\frac{\alpha^{\prime} E}{m \rho_{0}}$. Performing the integral, with a proper regularization, one finds

$$
\left(n+\frac{1}{2}\right) \pi=-\frac{\sqrt{\pi}}{2 z} \frac{\Gamma\left(\frac{d-2 \theta}{d(z+1)-2 \theta}\right)}{\Gamma\left(\frac{1}{2}+\frac{d-2 \theta}{d(z+1)-2 \theta}\right)}\left(\frac{\alpha^{\prime} E}{m \rho_{0}}\right)^{\frac{z d}{2 \theta-z d-d}} E .
$$

It is worth to note that, unlike the $\mathrm{AdS}$ case, in the present case due to the non-trivial IR geometry, the string while pulsating cannot approach $r=0$. Indeed 
there is a low bound for the string in the radial direction fixed by

$$
r \geq\left(\frac{m \rho_{0}}{E \alpha^{\prime}}\right)^{\frac{d}{d+d z-2 \theta}}
$$

\section{Discussions}

In this paper we have studied certain features of strongly coupled theories with hyperscaling violation by making use of AdS/CFT correspondence. More precisely we have considered a gravitational model on a background which is conformally a metric with the Lifshitz isometry. The metric has two parameters corresponding to dynamical and hyperscaling violation exponents. This geometry may be found from an Einstein-Maxwell-Dilaton theory.

By making use of a double Wick rotation it is possible to have a situation where the anisotropic scaling could be along either time or one of spatial directions. In both cases, using AdS/CFT correspondence, we have computed several quantities including holographic entanglement entropy, Wilson loop, drag force as well as energy loss of a rotating string.

Although we have studied the different quantities for a generic dynamical and hyperscaling violation exponents in arbitrary dimensions, we have found that for particular values of the parameters the theory exhibits interesting behavior.

To compute the entanglement entropy for a strip in a theory with an anisotropic scaling along a spatial direction we considered two distinctive cases depending on whether the width of the strip is along the anisotropic direction. When the width of the strip is along the anisotropic direction we have found that when $\theta=d-1$ for any $z$, the entanglement entropy exhibits a logarithmic violation of area law while for $\theta=d$, the system has an extensive entropy. Indeed the situation is very similar to the case where the anisotropic scaling is along the time direction [20]. On the other hand for the case where the width of strip is along a spatial direction with a normal scaling, the entanglement entropy shows logarithmic behavior for $\theta=d$ and $z=2$, while in this case the subsystem never has an extensive entropy. Having found the logarithmic behavior might indicate that the model has a Fermi surface.

For an open string probing the geometry one can read several interesting quantities, such as Wilson loop which in turn can be used to read the effective potential between two external point like objects in the system. We have shown that typically the effective potential is power low, though for special cases it could be logarithmic.

For a moving or rotating open string we have observed that even though the geometry are at zero temperature, the induced metric on the worldvolume of the open string has an event horizon leading to a non-zero Hawking termperature for worldvoulme theory. This might be the reason we are getting non-zero drag force for the string. Again in this case we find that $\theta=d$ is an special case where the string shows an anomalous behavior.

We have also probed the background with different closed strings. The closed 
strings we have considered include rotating folded and circular pulsating strings. From the gravity point of view it is possible to find the energy of the strings in terms of the quantum numbers the strings may have. This may be used to find the dispersion relation of a possible field configuration in the dual strongly coupled hyperscaling violation field theory. The anomalous behavior can also been seen for closed string too. In particular for $z d=2 \theta$ ( which includes $\theta=d, z=2$ ) the dispersion relation is logarithmic.

In this paper we have only considered a zero temperature background, though it could be generalized to the finite temperature too. In fact the finite temperature metric has the following form [20]

$$
d s_{d+2}^{2}=\left(\frac{r}{r_{F}}\right)^{\frac{2 \theta}{d}}\left(-r^{-2(z-1)} f(r) d t^{2}+\frac{d r^{2}}{f(r)}+d x_{i}^{2}\right)
$$

where

$$
f(r)=1-\frac{r^{d+z-\theta}}{r_{h}^{d+z-\theta}}
$$

It would be interesting to study the quantities we have considered in this paper at finite temperature.

\section{Acknowledgments}

We would like to thank Eoin Ó Colgáin, Mohammad Mohammadi Mozaffar, Ali Mollabashi, Amir E. Mosaffa and Ali Vahedi for discussions on different aspects of holographic entanglement entropy and hyperscaling violation theories. The research of M.A. is supported by Iran National Science Foundation (INSF). The work of H.Y. is supported by the National Research Foundation of Korea Grant funded by the Korean Government (NRF-2011- 0023230)

\section{References}

[1] J. M. Maldacena, "The Large N limit of superconformal field theories and supergravity," Adv. Theor. Math. Phys. 2, 231 (1998) [Int. J. Theor. Phys. 38, 1113 (1999)] hep-th/9711200.

[2] S. S. Gubser, I. R. Klebanov and A. M. Polyakov, "Gauge theory correlators from noncritical string theory," Phys. Lett. B 428, 105 (1998) hep-th/9802109].

[3] E. Witten, "Anti-de Sitter space and holography," Adv. Theor. Math. Phys. 2, 253 (1998) hep-th/9802150.

[4] S. A. Hartnoll, "Lectures on holographic methods for condensed matter physics," Class. Quant. Grav. 26, 224002 (2009) arXiv:0903.3246 [hep-th]]. 
[5] S. Kachru, X. Liu and M. Mulligan, "Gravity duals of Lifshitz-like Fixed Points," Phys. Rev. D78 (2008) 106005, arXiv:0808.1725.

[6] P. Koroteev and M. Libanov, "On Existence of Self-Tuning Solutions in Static Braneworlds without Singularities," JHEP 0802, 104 (2008) arXiv:0712.1136 [hep-th]].

[7] M. Taylor, "Non-relativistic holography," arXiv:0812.0530.

[8] D. T. Son, "Toward an AdS/cold atoms correspondence: A Geometric realization of the Schrodinger symmetry," Phys. Rev. D 78, 046003 (2008) arXiv:0804.3972 [hep-th]];

[9] K. Balasubramanian and J. McGreevy, "Gravity duals for non-relativistic CFTs," Phys. Rev. Lett. 101, 061601 (2008) [arXiv:0804.4053 [hep-th]].

[10] M. Alishahiha and O. J. Ganor, "Twisted backgrounds, PP waves and nonlocal field theories," JHEP 0303, 006 (2003) hep-th/0301080.

[11] E. Perlmutter, "Domain Wall Holography for Finite Temperature Scaling Solutions," JHEP 1102, 013 (2011) arXiv:1006.2124 [hep-th]].

[12] G. Bertoldi, B. A. Burrington and A. W. Peet, "Thermal behavior of charged dilatonic black branes in AdS and UV completions of Lifshitz-like geometries," Phys. Rev. D 82, 106013 (2010) arXiv:1007.1464 [hep-th]].

[13] G. Bertoldi, B. A. Burrington, A. W. Peet and I. G. Zadeh, "Lifshitz-like black brane thermodynamics in higher dimensions," Phys. Rev. D 83, 126006 (2011) arXiv:1101.1980 [hep-th]].

[14] K. Goldstein, N. Iizuka, S. Kachru, S. Prakash, S. P. Trivedi and A. Westphal, "Holography of Dyonic Dilaton Black Branes," JHEP 1010, 027 (2010) [arXiv:1007.2490 [hep-th]].

[15] N. Iizuka, N. Kundu, P. Narayan and S. P. Trivedi, "Holographic Fermi and Non-Fermi Liquids with Transitions in Dilaton Gravity," arXiv:1105.1162 [hepth].

[16] P. Berglund, J. Bhattacharyya and D. Mattingly, "Charged Dilatonic AdS Black Branes in Arbitrary Dimensions," arXiv:1107.3096 [hep-th].

[17] C. Charmousis, B. Gouteraux, B. S. Kim, E. Kiritsis and R. Meyer, "Effective Holographic Theories for low-temperature condensed matter systems," JHEP 1011, 151 (2010) arXiv:1005.4690 [hep-th]].

[18] B. Gouteraux and E. Kiritsis, "Generalized Holographic Quantum Criticality at Finite Density," JHEP 1112 (2011) 036 [arXiv:1107.2116 [hep-th]]. 
[19] L. Huijse, S. Sachdev and B. Swingle, "Hidden Fermi surfaces in compressible states of gauge-gravity duality," arXiv:1112.0573 [cond-mat.str-el].

[20] X. Dong, S. Harrison, S. Kachru, G. Torroba and H. Wang, "Aspects of holography for theories with hyperscaling violation," JHEP 1206, 041 (2012) arXiv:1201.1905 [hep-th]].

[21] N. Ogawa, T. Takayanagi and T. Ugajin, "Holographic Fermi Surfaces and Entanglement Entropy," arXiv:1111.1023 [hep-th].

[22] J. Bhattacharya, S. Cremonini and A. Sinkovics, "On the IR completion of geometries with hyperscaling violation," arXiv:1208.1752 [hep-th].

[23] N. Kundu, P. Narayan, N. Sircar and S. P. Trivedi, "Entangled Dilaton Dyons," arXiv:1208.2008 [hep-th].

[24] S. Ryu and T. Takayanagi, "Holographic Derivation of Entanglement Entropy from AdS/CFT," Phys. Rev. Lett. 96 (2006) 181602 [hep-th/0603001].

[25] S. Ryu and T. Takayanagi, "Aspects of Holographic Entanglement Entropy," JHEP 0608 (2006) 045 hep-th/0605073.

[26] J. de Boer, M. Kulaxizi and A. Parnachev, "Holographic Entanglement Entropy in Lovelock Gravities," JHEP 1107, 109 (2011) [arXiv:1101.5781 [hep-th]].

[27] S. -J. Rey and J. -T. Yee, "Macroscopic strings as heavy quarks in large N gauge theory and anti-de Sitter supergravity," Eur. Phys. J. C 22, 379 (2001) [hep-th/9803001].

[28] J. M. Maldacena, "Wilson loops in large N field theories," Phys. Rev. Lett. 80, 4859 (1998) hep-th/9803002.

[29] S. S. Gubser, "Drag force in AdS/CFT," Phys. Rev. D 74, 126005 (2006) hepth/0605182.

[30] E. Kiritsis, "Lorentz violation, Gravity, Dissipation and Holography," arXiv:1207.2325 [hep-th].

[31] A. Akhavan, M. Alishahiha, A. Davody and A. Vahedi, "Non-relativistic CFT and Semi-classical Strings," JHEP 0903, 053 (2009) [arXiv:0811.3067[hep-th]].

[32] K. B. Fadafan, "Drag force in asymptotically Lifshitz spacetimes," arXiv:0912.4873 [hep-th].

[33] U. Gursoy, E. Kiritsis, L. Mazzanti and F. Nitti, "Langevin diffusion of heavy quarks in non-conformal holographic backgrounds," JHEP 1012, 088 (2010) arXiv:1006.3261 [hep-th]]. 
[34] K. B. Fadafan, H. Liu, K. Rajagopal and U. A. Wiedemann, "Stirring Strongly Coupled Plasma," Eur. Phys. J. C 61 (2009) 553 [arXiv:0809.2869 [hep-ph]].

[35] C. Athanasiou, P. M. Chesler, H. Liu, D. Nickel and K. Rajagopal, "Synchrotron radiation in strongly coupled conformal field theories," Phys. Rev. D 81, 126001 (2010) [Erratum-ibid. D 84, 069901 (2011)] [arXiv:1001.3880 [hep-th]].

[36] K. B. Fadafan and H. Soltanpanahi, "Energy loss in a strongly coupled anisotropic plasma," arXiv:1206.2271 [hep-th].

[37] M. Ali-Akbari and U. Gursoy, "Rotating strings and energy loss in nonconformal holography," JHEP 1201, 105 (2012) [arXiv:1110.5881 [hep-th]].

[38] K. Narayan, "On Lifshitz scaling and hyperscaling violation in string theory," arXiv:1202.5935 [hep-th].

[39] M. Ammon, M. Kaminski and A. Karch, "Hyperscaling-Violation on Probe D-Branes," arXiv:1207.1726 [hep-th].

[40] S. S. Gubser, I. R. Klebanov and A. M. Polyakov, "A Semiclassical limit of the gauge / string correspondence," Nucl. Phys. B 636, 99 (2002) [hep-th/0204051].

[41] J. A. Minahan, "Circular semiclassical string solutions on AdS(5) x S(5)," Nucl. Phys. B 648 (2003) 203 hep-th/0209047.

[42] M. Alishahiha and A. E. Mosaffa, "Circular semiclassical string solutions on confining AdS / CFT backgrounds," JHEP 0210, 060 (2002) [hep-th/0210122]. 